CASE REPORT

\title{
Nosocomial COVID-19 infection mortality following surgery for severe progressive cervical myelopathy: a case report
}

\author{
Andrew S. Zhang ${ }^{1 凶}$, Rodrigo Saad Berreta ${ }^{1}{ }^{1}$, Camilo Osorio ${ }^{1}$ and Alan H. Daniels ${ }^{1}$ \\ (c) The Author(s), under exclusive licence to International Spinal Cord Society 2021
}

\begin{abstract}
INTRODUCTION: We present the unique case of a nosocomial COVID infection acquired after urgent surgical intervention for cervical myelopathy, as well as the sequelae that followed in the postoperative period.

CASE PRESENTATION: An initially COVID-negative patient underwent urgent surgical intervention for cervical myelopathy with significant neurological deterioration. She underwent an uncomplicated staged anterior cervical discectomy and fusion with corpectomy, as well as a subsequent posterior cervical instrumented fusion within the same hospitalization. The patient would refuse to adhere to standard COVID precautions during her admission and demonstrated rapid decompensation following her particularly uneventful surgeries, ultimately leading to her expiration. A laboratory test confirmed that she had contracted COVID at the time of the patient's death.

DISCUSSION: This report highlights the repercussions of COVID-19 infection during the perioperative period and its implications on surgical outcomes. The stresses of surgery and the body's immunosuppressive responses during this time place patients at particular risk for the contraction of this virus. The standard precautions should be followed and vaccination should be considered for surgical candidates prior to their operations, as they become more readily accessible.
\end{abstract}

Spinal Cord Series and Cases (2021)7:100; https://doi.org/10.1038/s41394-021-00465-8

\section{INTRODUCTION}

The novel coronavirus 2019 (COVID-19) has pervaded all aspects of life. Healthcare systems have had to adapt accordingly to prevent nosocomial transmission, mitigate potential infection of healthcare workers, and conserve medical resources such as ICU beds and ventilators. Within the field of spine surgery, certain strategies have been effected since the onset of this pandemic to meet the demands of this new reality, such as deferring elective surgeries, implementing mandatory preoperative testing, and stratifying types of surgery based on available resources [1-3]. Additionally, proceeding with surgery in confirmed COVID-positive patients has demonstrated worse outcomes and increased morbidity and mortality [4-7]. Yet, surgical emergencies often prompt dilemmas in decision-making in already-difficult times such as these. We describe the case of the management of a patient with progressive severe cervical myelopathy in the setting of a surge in active COVID cases nationwide.

\section{Case presentation}

A 66-year-old female with a past medical history significant for diabetes mellitus complicated by retinopathy, hypertension, hyperlipidemia, and B12 deficiency, presented to the emergency room with frequent falls, worsening balance and new-onset urinary incontinence, rapidly deteriorating over the past three months. On examination, she demonstrated full sensation and strength to her bilateral upper and lower extremities. She was hyperreflexic and ataxic. Imaging revealed multilevel degenerative changes within the cervical spine, with focal kyphosis at C5-6, and large disc herniations at C5-6 and C6-7 causing effacement of the cord, severe central and foraminal stenosis, with the associated signal change of the cord at the C5-6 level (Fig. 1).

Because of the rapid progression of her symptoms and urinary incontinence, the decision was made to proceed with urgent operative intervention. At the time of her visit to the emergency department (ED), she was noted to have tested negative for SARSCoV-2 via a nasopharyngeal polymerase chain reaction (PCR) test. During her admission, she also ardently refused to wear a mask despite being asked to do so on multiple instances. She did have another routine test performed 11 days later due to her prolonged stay, which was also negative. Based on her pathology, the decision was made to proceed with a staged circumferential decompression and fusion. On the first surgical date, 6 days following her initial ED visit, she would undergo a C5-7 anterior cervical discectomy and fusion with complete C6 corpectomy and cage reconstruction (Fig. 2). This would allow us to achieve aggressive undercutting of C5 and C7 posterior osteophytes. The operation was uncomplicated and expeditious, there were no changes with neuromonitoring throughout the procedure and she was able to be successfully extubated without issue. She was placed into a hard collar postoperatively with a plan to return to the operating room (OR) 2 days later, for which she underwent C3$\mathrm{T} 1$ posterior cervical instrumented fusion with posterior column osteotomies at C3-4 and C4-5 to help correct her fixed spondylolisthesis (Fig. 3). Again, neuromonitoring was stable throughout the procedure and she was able to be safely extubated upon the conclusion of the case.

${ }^{1}$ Department of Orthopaedic Surgery, Brown University Warren Alpert Medical School, Providence, RI, USA. ${ }^{\varpi_{e}}$ mail: Andrew_zhang4@brown.edu 


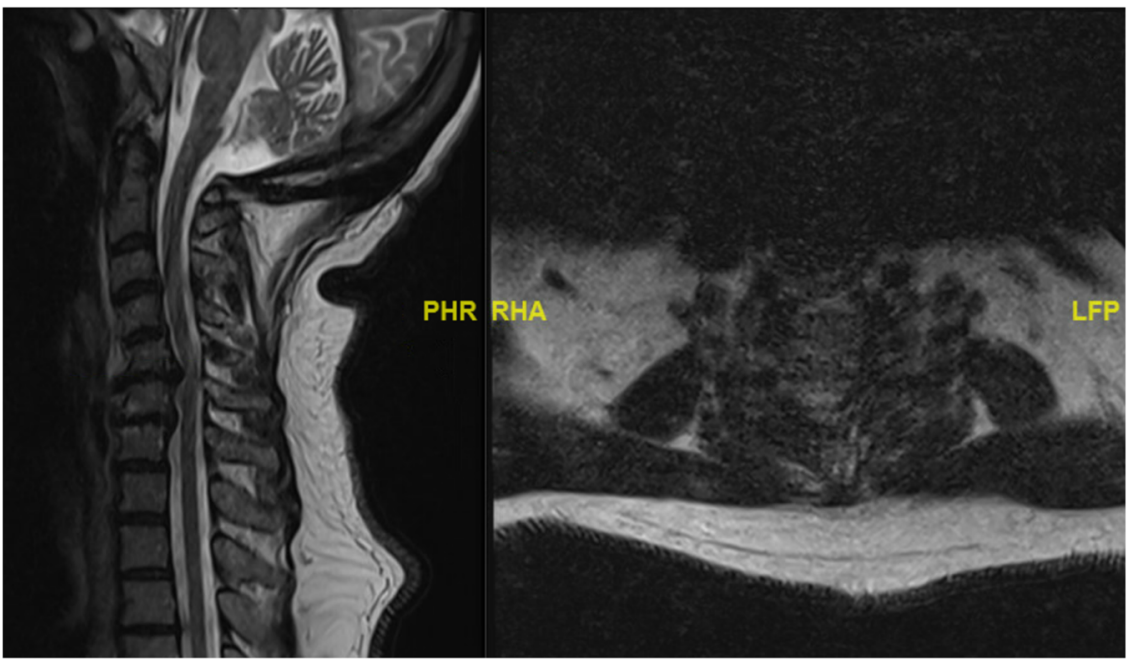

(a)

(b)

Fig. 1 Preoperative magnetic resonance imaging (MRI) study of the patient's cervical spine. a Midsagittal and $\mathbf{b}$ axial cuts at the C5-6 level of the T2-weighted MRI of the cervical spine, demonstrating multilevel degenerative changes, disc herniations at C5-6 and C6-7 causing severe central and foraminal stenosis with cord signal changes.

Postoperatively, the patient was admitted to the surgical intensive care unit (SICU) for a higher level of monitoring. Prior to being discharged to the floor, the patient did require supplemental oxygen via nasal cannula and was in sinus tachycardia. She also became episodically confused and disoriented. However, she was still deemed medically stable to be discharged to the floor by the medical team. Upon spine surgery evaluation on Postoperative Day 5, the patient became unresponsive during the examination and a rapid response was called. She became responsive shortly thereafter. Labs obtained at that time demonstrated a leukocytosis of $24.7 \times 10^{9} / \mathrm{L}$, a glucose of $205 \mathrm{mg} /$ $\mathrm{dL}$, and a C-reactive protein of $92.75 \mathrm{mg} / \mathrm{L}$. Repeat MRI was obtained and did not demonstrate any evidence of cord compression and expected postoperative seroma/hematoma. A repeat COVID PCR test was administered. Blood cultures were obtained. Empiric Vancomycin was started to treat Systemic Inflammatory Response Syndrome. Her repeat COVID test returned positive and recommendations by the infectious disease team to start Remdesivir were made on Postoperative Day 6. However, later that same day, the patient again became unresponsive and a Code Blue was initiated. Cardiopulmonary resuscitation was attempted and intubation was performed but was unable to be resuscitated and expired.

\section{DISCUSSION}

The implications of this patient's death in the setting of COVID are profound, not only for spine surgery but may be extrapolated to all invasive procedures in general. The patient was COVID negative upon initial presentation in the ED and again later during her hospital stay, but ultimately did test positive shortly before on the day of her demise. Of note, she did staunchly refuse standard precautions such as donning a mask during her hospitalization. Given this change in COVID status, the idea of a nosocomial infection must be suspected. Nevertheless, the patient expired within a few days following surgery, with new-onset symptoms including leukocytosis, altered mental status, hypoxia, urinary retention, and tachycardia. With a high WBC count of $>24$, the differential consisting of aspiration pneumonia, a urinary tract infection, COVID, or possible a Clostridium difficile infection responsible for the etiology of these symptoms, relevant workups were performed, with a COVID positive test returning at the time of expiration. If this nosocomial contraction of COVID was responsible for this patient's death, are there more measures that should have been taken to prevent this morbidity?

\section{COVID-19 on surgical outcomes/morbidity/mortality}

The effect of COVID-19 on surgical outcomes within orthopedic and neurological surgery has already been well described. Early reports have shown that patients with confirmed perioperative COVID-19 infections have higher mortality rates, intensive care unit (ICU) admissions, and postoperative complications [4-7]. Kayani et al. showed that patients who were undergoing urgent procedures to repair severe injuries to the musculoskeletal system, such as hip fractures, experience mortality rates of $30.5 \%$ in COVID-19 positive patients compared to $10.5 \%$ in controls [7]. As expected, patients with COVID-19 are also at high risk for pulmonary complications, placing them at elevated risk for ICUlevel care. Postoperative pulmonary complications such as pneumonia, acute respiratory distress syndrome (ARDS), or unexpected use of a ventilator and occurred in approximately $50 \%$ of patients with perioperative SARS-CoV-2 infections [4]. The high incidence of postoperative ventilation requirement in COVID19 patients can further damage the musculoskeletal system. Mechanical ventilation can provoke systemic inflammation and also may augment cases of ARDS seen in COVID-19 infections $[8,9]$. Moreover, prolonged use of ventilators can lead to peripheral and respiratory muscle atrophy due to the activation of protein degradation pathways as a result of oxidative stress $[10,11]$.

Surgery can also induce immunosuppressive responses in addition to proinflammatory conditions in postoperative patients, making them particularly susceptible to COVID-19 infections [12]. Therefore, considerable inflammatory stress from both COVID-19 infections and major surgery can create a synergetic effect [12] resulting in compounded neurologic, muscle, bone, and joint impairment [13]. Although this patient did not comply with precautions such as wearing personal protective equipment (PPE), her urgent surgery may have innately predisposed her to a weakened immune system, placing her at higher risk for contracting COVID-19. It's unclear when this infection was transmitted to her and whether she could have prevented this presumed nosocomial infection had she maintained these measures. However, becoming COVID-19 positive at some point during the perioperative period certainly placed her at much higher risk factors for a poor outcome. 


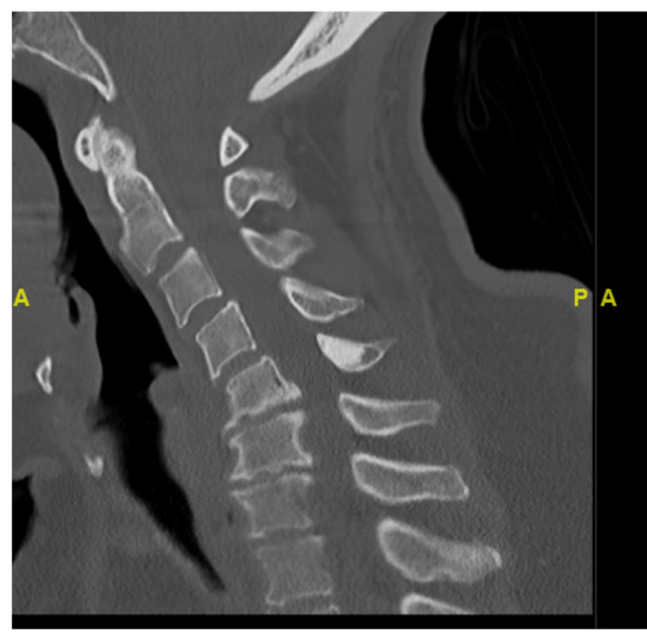

(a)

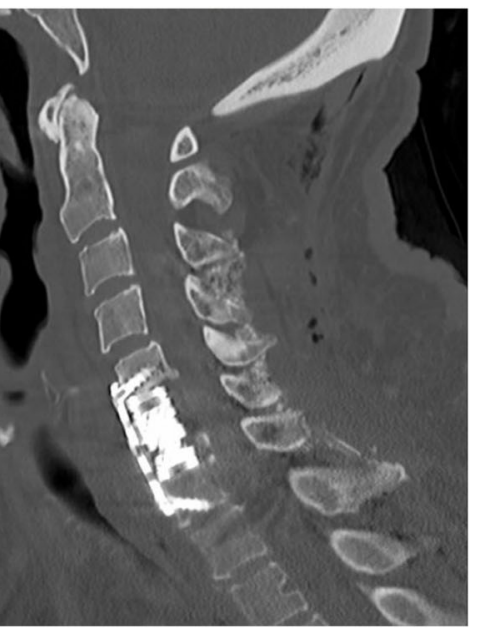

(b)

Fig. 2 Computed tomography (CT) scans of the patient's cervical spine. a Preoperative and b postoperative midsagittal CT scans of the cervical spine following first stage C6 corpectomy with cage reconstruction and C5-7 ACDF.

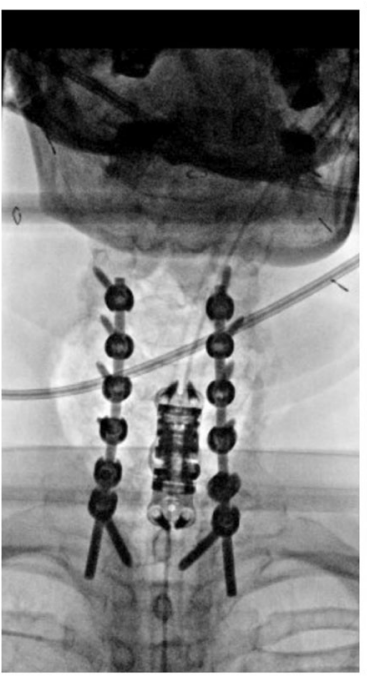

(a)

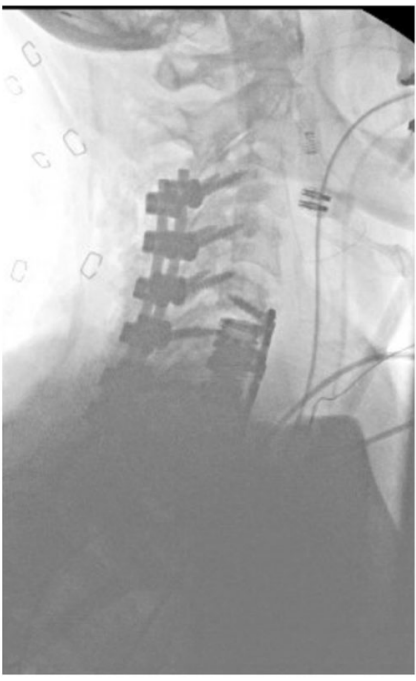

(b)
Fig. 3 Intraoperative fluoroscopic images after final surgery. Intraoperative posteroanterior $\mathbf{a}$ and lateral $\mathbf{b}$ fluoroscopic images following second-stage surgery demonstrating C6 corpectomy with C5-7 ACDF and C3-T1 posterior cervical instrumented fusion.

\section{Vaccination and its role in the perioperative period}

The development of vaccinations has revolutionized public health. Most vaccines are aimed to provide prophylactic protection, generating an immune response within the host, which creates neutralizing antibodies that prevent viral entry into host cells [14]. At the time of publication of this article, two vaccinations have been authorized and recommended by the Centers of Disease Control and Prevention (CDC), including the Pfizer-BioNTech COVID-19 vaccine and the Moderna COVID-19 vaccine [15]. The Pfizer-BioNTech and Moderna vaccines have shown a protective effect of $95 \%$ and $94.1 \%$, respectively, with an additional reduction of almost $100 \%$ in cases of severe COVID-19 $[16,17]$. While still in the infancy of these vaccines, there remains a paucity of evidence of the protective role these vaccines may impart on surgical patients as widespread rollout to the public has yet to commence. Vaccination may certainly become a prerequisite before elective surgeries are even scheduled in the near future, but their utility may still be dismissed during times of trauma and emergencies.

\section{When should spine surgery be delayed?}

Recommendations put forth by institutions such as the American Academy of Orthopedic Surgeons, the American College of Surgeons, and the North American Spine Society, as well as several prominent experts within the realm of spine surgery both within orthopedic surgery and neurosurgery have suggested that this patient's progressive cervical myelopathy with neurologic deterioration prompting Emergency Room evaluation to be an "Emergent" or "Urgent" condition, to be addressed without postponement [18-24]. Given these recommendations, the decision was made to proceed in order to curtail any further neurological deficits. Although this case report illustrates the grave risk of proceeding with spine operations during the pandemic, we believe that the surgery should not have been delayed and elect to present this case as a cautionary tale for patients who require surgery during these times. In this case, the patient actively violated the protocols set in place for her protection in refusing to donn a mask during her hospitalization. Studies have shown that physically distancing, wearing eye shields, and using face masks have considerable effects in stunting viral transmission rates, with a greater reduction in risk seen in N95 masks [25]. Considering the immunosuppressive and proinflammatory conditions present in postoperative patients [12], adhering to hospital guidelines is integral to avoid complications and nosocomial COVID-19 infections. To prevent further instances, it may be necessary to integrate patient education in the treatment course and recovery of urgent orthopedic procedures. In cases of noncompliant patients, physicians may ultimately choose to delay procedures, empowered by this substantial body of evidence toward poor postoperative outcomes, and extensively counseling patients of the risks associated with surgery during the COVID-19 pandemic.

\section{CONCLUSION}

Much remains unknown about the repercussions of COVID-19 infection during the perioperative period and its implications on surgical outcomes. Manifestations of this potentially devastating respiratory disease are highly variable and precautions should be followed. The role of vaccination remains to be seen and may be 
considered for surgical candidates prior to their operations, namely in the outpatient setting.

\section{REFERENCES}

1. Massey PA, McClary K, Zhang AS, Savoie FH, Barton RS. Orthopaedic surgical selection and inpatient paradigms during the coronavirus (COVID-19) pandemic. J Am Acad Orthop Surg. 2020;28(Jun):436-50. https://doi.org/10.5435/JAAOS-D-20-00360.

2. Zhang AS, Myers M, Kee CJ, McClary KN, Barton RS, Massey PA. Adapting orthopaedic surgery training programs during the COVID-19 pandemic and future directions. Arthrosc Sports Med Rehabil. 2020;2(Oct):e683-e696. https:// doi.org/10.1016/j.asmr.2020.06.008.

3. Rodrigues-Pinto R, Sousa R, Oliveira A. Preparing to perform trauma and orthopaedic surgery on patients with COVID-19. J Bone Joint Surg Am. 2020;102 (Jun):946-50. https://doi.org/10.2106/JBJS.20.00454.

4. COVIDSurg Collaborative. Mortality and pulmonary complications in patients undergoing surgery with perioperative SARS-CoV-2 infection: an international cohort study. Lancet. 2020;396(Jul):27-38. https://doi.org/10.1016/S0140-6736(20)31182-X.

5. Doglietto F, Vezzoli M, Gheza F, Lussardi GL, Domenicucci M, Vecchiarelli L, et al. Factors associated with surgical mortality and complications among patients with and without coronavirus disease 2019 (COVID-19) in Italy. JAMA Surg. 2020;155 (Jun):1-14. https://doi.org/10.1001/jamasurg.2020.2713.

6. Knisely A, Zhou ZN, Wu J, Huang Y, Holcomb K, Melamed A, et al. Perioperative morbidity and mortality of patients with COVID-19 who undergo urgent and emergent surgical procedures. Ann Surg. 2021;273(Jan):34-40. https://doi.org/ 10.1097/SLA.0000000000004420.

7. Kayani B, Onochie E, Patil V, Begum F, Cuthbert R, Ferguson D, et al. The effects of COVID-19 on perioperative morbidity and mortality in patients with hip fractures. Bone Joint J. 2020;102-B(Sep):1136-45. https://doi.org/10.1302/0301-620X.102B9. BJJ-2020-1127.R1.

8. Ranieri VM, Suter PM, Tortorella C, De Tullio R, Dayer JM, Brienza A, et al. Effect of mechanical ventilation on inflammatory mediators in patients with acute respiratory distress syndrome: a randomized controlled trial. JAMA. 1999;282 (Jul):54-61. https://doi.org/10.1001/jama.282.1.54.

9. Dhanireddy S, Altemeier WA, Matute-Bello G, O'Mahony DS, Glenny RW, Martin $\mathrm{TR}$, et al. Mechanical ventilation induces inflammation, lung injury, and extrapulmonary organ dysfunction in experimental pneumonia. Lab Invest. 2006;86 (Aug):790-9. https://doi.org/10.1038/labinvest.3700440.

10. Tobin MJ, Laghi F, Jubran A. Narrative review: ventilator-induced respiratory muscle weakness. Ann Intern Med. 2010;153(Aug):240-5. https://doi.org/10.7326/ 0003-4819-153-4-201008170-00006.

11. Moura $P$, Ferreira $P$, Luna $M$, Silva Junior $I$, Duarte $P$, Reinaux $C$, et al. Profile of the peripheral muscular atrophy during the first days of mechanical ventilation: a longitudinal ultrasonographic study. Eur Resp J. 2019;54(Sep) Suppl 63:PA4834. https://doi.org/10.1183/13993003.congress-2019.PA4834.

12. Besnier E, Tuech JJ, Schwarz L. We asked the experts: Covid-19 outbreak: is there still a place for scheduled surgery? "Reflection from pathophysiological data". World J Surg. 2020;44(Jun):1695-8. https://doi.org/10.1007/s00268-020-05501-6.

13. Disser NP, De Micheli AJ, Schonk MM, Konnaris MA, Piacentini AN, Edon DL, et al. Musculoskeletal consequences of COVID-19. J Bone Joint Surg Am. 2020;102 (Jul):1197-204. https://doi.org/10.2106/JBJS.20.00847.

14. Patel SS, Kalma J, Bluman EM. Understanding COVID-19 vaccines and their development. J Bone Joint Surg Am. 2020;102:1759-69. https://doi.org/10.2106/ JBJS.20.01191.

15. World Health Organization. Draft landscape of COVID-19 candidate vaccines. 2020. https://www.who.int/who-documents-detail/draft-landscape-of-covid-19candidate-vaccines. Accessed 20 May 2021.

16. Polack FP, Thomas SJ, Kitchin N, Absalon J, Gurtman A, Lockhart S, et al. Safety and efficacy of the BNT162b2 mRNA Covid-19 vaccine. N Engl J Med. 2020;383:2603-15. https://doi.org/10.1056/NEJMoa2034577
17. Baden LR, El Sahly HM, Essink B, Kotloff K, Frey S, Novak R, et al. Efficacy and safety of the mRNA-1273 SARS-CoV-2 vaccine. N Engl J Med. 2021;384 (Feb):403-16. https://doi.org/10.1056/NEJMoa2035389.

18. DeKeyser GJ, Brodke DS, Saltzman CL, Lawrence BD. Response to the coronavirus disease 2019 pandemic by the spine division at a Level-I academic referral center. J Am Acad Orthop Surg. 2020;28(Dec):1003-8. https://doi.org/10.5435/JAAOS-D20-00493.

19. Guy DK. AAOS guidelines for elective surgery during the COVID-19 pandemic. American Academy of Orthopaedic Surgeons web. 2020. https://www.aaosorg/ about/covid-19-information-for-our-members/aaos-guidelines-for-electivesurgery/. Accessed 20 May 2021.

20. COVID-19: elective case triage guidelines for surgical care. American College of Surgeons. 2020. www.facs.org/covid-19/clinical-guidance/elective-case. Accessed 20 May 2021.

21. Parvizi J, Gehrke T, Krueger CA, Chisari E, Citak M, Van Onsem S, et al. Resuming elective orthopaedic surgery during the COVID-19 pandemic: guidelines developed by the International Consensus Group (ICM). J Bone Joint Surg Am. 2020;102(Jul):1205-12. https://doi.org/10.2106/JBJS.20.00844

22. Awad ME, Rumley JCL, Vazquez JA, Devine JG. Perioperative considerations in urgent surgical care of suspected and confirmed COVID-19 orthopaedic patients: operating room protocols and recommendations in the current COVID-19 pandemic. J Am Acad Orthop Surg. 2020;28(Jun):451-63. https://doi.org/10.5435/ JAAOS-D-20-00227.

23. Donnally CJ III, Shenoy K, Vaccaro AR, Schroeder GD, Kepler CK. Triaging spine surgery in the COVID-19 era. Clin Spine Surg. 2020;33(May):129-30.

24. Burke JF, Chan AK, Mummaneni V, Chou D, Lobo EP, Berger MS, et al. Letter: the coronavirus disease 2019 global pandemic: a neurosurgical treatment algorithm. Neurosurgery. 2020;87(Jul):E50-E56. https://doi.org/10.1093/ neuros/nyaa116.

25. Chu DK, AkI EA, Duda S, Solo K, Yaacoub S, Schünemann HJ. Physical distancing, face masks, and eye protection to prevent person-to-person transmission of SARS-CoV-2 and COVID-19: a systematic review and meta-analysis. Lancet. 2020;395(Jun):1973-87. https://doi.org/10.1016/S0140-6736(20)31142-9.

\section{AUTHOR CONTRIBUTIONS}

ASZ and AHD contributed to the conceptualization of this project. All authors contributed equally to the literature review, writing and revisions of this manuscript.

\section{COMPETING INTERESTS}

ASZ, RSB, and CO do not possess any potential conflicts of interest. AHD reports disclosures as follows: EOS, paid consultant; Orthofix, Inc., paid consultant, research support; SpineArt, paid consultant; Springer: publishing royalties, financial or material support; Stryker: paid consultant, all outside submitted work.

\section{ADDITIONAL INFORMATION}

Supplementary information The online version contains supplementary material available at https://doi.org/10.1038/s41394-021-00465-8.

Correspondence and requests for materials should be addressed to Andrew S. Zhang.

Reprints and permission information is available at http://www.nature.com/ reprints

Publisher's note Springer Nature remains neutral with regard to jurisdictional claims in published maps and institutional affiliations. 